\title{
Image compression algorithm of floral canopy based on mask hybrid coding for ROI
}

\author{
Sun Guoxiang, Wang Xiaochan*, Ding Yongqian, Li Yuhua, \\ Zhang Baohua, Li Yongbo, Zhang Yu \\ (College of Engineering, Nanjing Agricultural University, Nanjing 210095, China)
}

\begin{abstract}
To achieve high-quality image compression of a floral canopy, a region of interest (ROI) mask of the wavelet domain was generated through the automatic identification of the canopy ROI and lifting the bit-plane of the ROI to obtain priority of coding for the ROI-set partitioning in hierarchical trees (ROI-SPIHT) coding. The embedded zerotree wavelet (EZW) coding was conducted for the background (BG) region of the image and a relatively more low-frequency wavelet coefficient was obtained using a relatively small amount of coding. Through the weighing factor $r$ of the ROI coding amount, the proportion of the ROI and BG coding amount was dynamically adjusted to generate embedded, truncatable bit streams. Despite the location of truncation, the image information and ROI mask information required by the decoder can be guaranteed to achieve high-quality compression and reconstruction of the image ROI. The results indicated that under the same bit rate, the larger the $r$ value is, the larger the peak-signal-to-noise ratio (PSNR) for the ROI reconstructed image and the smaller the $P S N R$ for the BG reconstructed image. In the range of 0.07-1.09 bpp, the PSNR of the ROI reconstructed image was $42.65 \%$ higher on average than that of the BG reconstructed image, $43.95 \%$ higher on average than that of the composite image of the ROI and BG (ALL), and 16.84\% higher on average than that of the standard SPIHT reconstructed image. Additionally, the mean square error of the quality evaluation index and similarity for the ROI reconstructed image were both better than those for the BG, ALL, and standard SPIHT reconstructed images. The texture distortion of the ALL image was smaller than that of the SPIHT reconstructed image, indicating that the image compression algorithm based on the mask hybrid coding for ROI (ROI-MHC) is capable of improving the reconstruction quality of an ROI image. When the weighing factor $r$ is a fixed value, as the proportion of ROI $(a)$ increases, the quality of ROI image reconstruction gradually decreases. Therefore, upon the application of the ROI-MHC image compression algorithm, high-quality reconstruction of the ROI image can be achieved through dynamically configuring $r$ according to $a$. Under the same bit rate, the quality of the ROI-MHC image compression is higher than that of current compression algorithms of same classes and offers promising application opportunities.
\end{abstract}

Keywords: floral canopy, ROI, mask hybrid coding, image compression, algorithm, wavelet transform DOI: $10.25165 /$ j.ijabe.20171005.2772

Citation: Sun G X, Wang X C, Ding Y Q, Li Y H, Zhang B H, Li Y B, et al. Image compression algorithm of floral canopy based on mask hybrid coding for ROI. Int J Agric \& Biol Eng, 2017; 10(5): 166-176.

\section{Introduction}

Plant growth information forms an important decisionmaking basis for modern intelligent facilities and

\section{Received date: 2016-08-11 Accepted date: 2017-07-31}

Biographies: Sun Guoxiang, $\mathrm{PhD}$, Lecturer, research interests: computer vision, and modeling biosystems, Email: sguoxiang@ njau.edu.cn; Ding Yongqian, PhD, Associate Professor, research interests: intelligent agricultural equipment, Email: yongqiand@ njau.edu.cn; Li Yuhua, PhD, Lecturer, research interests: early detection and diagnosis of plant disease, Email: lyhresearch@ njau.edu.cn; Zhang Baohua, $\mathrm{PhD}$, Lecturer, research interests: computer vision and spectral imaging, Email: bhzhang@ njau.edu.cn; Li Yongbo, PhD, Associate Professor, research horticulture control systems to achieve precise control ${ }^{[1]}$. These settings require the collection of a large amount of plant images. The collected images include plants and background $(\mathrm{BG})$ along with a considerable amount of

interests: modeling, biological systems and environment, Email: bewit@njau.edu.cn; Zhang Yu, PhD, research interests: machine vision and modeling biosystems, Email: Zhangyu64@hotmail.com. *Corresponding author: Wang Xiaochan, $\mathrm{PhD}$, Professor, research interests: modeling, biological systems and environment, Department of Electrical Engineering, College of Engineering, Nanjing Agricultural University, Box 96, No.40 Dianjiangtai Road, Pukou, Nanjing 210095, China. Tel: +86-25- 58606567, Email: wangxiaochan@njau.edu.cn. 
extraneous information. Therefore, the systems with limited bandwidth or capacity need to perform high-quality image compression. Region of interest (ROI) coding can be used to address the tradeoff between image quality and compression ratio in the application of image compression and achieve local ROI reconstruction with high resolution under the condition of low bit rate transmission $^{[2,3]}$. ROI coding has a broad scope of applications in detection using unmanned aerial vehicles $^{[4]}$, medical imaging ${ }^{[5]}$, and wireless image transmission with limited bandwidth ${ }^{[2,3]}$, among others.

At present, the main technical issue to be solved for the ROI coding of plant images is the algorithm of ROI identification and ROI coding/decoding ${ }^{[2]}$. Many studies have been performed on the ROI identification of plant images; these generally achieve precise segmentation of the plant region according to the color features of plants. The adopted methods mainly include the normalized difference index (NDI) proposed by Perez-Jimenez et $\mathrm{al}^{\left[{ }^{[6]}\right.}$, the normalized green-red difference index (NGRDI) proposed by Hunt et al. ${ }^{[7]}$, the excess green minus excess red (ExG-ExR) index proposed by Meyer et al. ${ }^{[8,9]}$, and the modified excess green minus excess red (MExG-ExR) segmentation algorithm of a floral canopy in a natural light environment proposed by Sun et al. ${ }^{[10,11]}$, which established the foundation for the ROI coding/decoding of plant images. There are two categories of ROI coding/decoding algorithms: standard shaped ROI coding and arbitrarily shaped ROI coding ${ }^{[2]}$. Specifically, standard shaped ROI coding mainly includes circular ROI coding $^{[12]}$ and rectangular ROI coding ${ }^{[13]}$, whereas arbitrarily shaped ROI coding mainly includes the normal displacement algorithm, maximum displacement algorithm, implicit ROI, and staggered displacement algorithm ${ }^{[2,14,15]}$. The key coding principle of these algorithms is to achieve accurate implementation of ROI priority coding using the scheme of lifting the ROI bit-plane. In particular, the normal displacement algorithm requires additional coding to describe the regional information of the ROI; the maximum displacement algorithm does not support the adjustment of the ROI priority level; the implicit ROI depends on the bit allocation algorithm of embedded block coding with optimized truncation (EBCOT) and post-compression rate-distortion optimization (OPT-PCRD) of the JPEG2000 format, and it is not universally applicable; and the staggered displacement algorithm adopts the method of staggered displacement to assign the ROI and BG coefficients to different bit-planes according to a predetermined scheme. This algorithm supports any arbitrary shape and provides an adjustable ROI priority level, but its disadvantage is the considerable amount of computation necessitated by the staggered processing of the coefficient bit-planes prior to coding. Moreover, because the shift of the wavelet coefficient with a staggered bit-plane occupies a relatively large bit width, the expense of storage is also relatively large for the embedded application ${ }^{[2,3,16,17]}$.

Therefore, in this study an image compression algorithm for a floral canopy was proposed based on mask hybrid coding for an ROI (abbreviated as ROI-MHC). This algorithm is based on an ROI mask to achieve the separation of the ROI and BG. The ROI obtains the priority coding right by lifting the ROI bit-plane, and ROI-set partitioning in a hierarchical tree (ROI-SPIHT) coding is conducted ${ }^{[2,18]}$; the embedded zerotree wavelet (EZW) coding was directly applied to the $\mathrm{BG}^{[19]}$, making it possible to acquire a relatively large number of low-frequency wavelet coefficients under the constraint of a relatively small amount of coding. As an improvement, the algorithm in this study introduced a weighing factor of the ROI coding amount and achieved dynamic adjustment of the proportion for the ROI and BG coding amounts under the condition of a fixed coding amount. Consequently, the ROI not only obtains priority coding but also can obtain more coding amounts. This algorithm supports multiple arbitrarily shaped ROI coding, an adjustable priority level of ROI coding, and adjustable allocation of the ROI and BG coding amounts. This algorithm generates embedded, truncatable bit streams and ensures image reconstruction by the decoder irrespective of the location of truncation, avoiding the additional encoding of the ROI mask after the image encoding; additionally, it is applicable for high-quality ROI compression in low-bit-rate applications and the 
requirement of ROI priority transmission in progressive transmission.

\section{Materials and methods}

The 'Jinchun No.3' cucumber variety was adopted to conduct the experiments in a Venlo-type greenhouse. A 3-megapixel half-sphere wide-angle webcam (manufactured by Hikvision, Model: DS-2CD7254F-E, Hangzhou, China) was installed above the flora canopy. The focal length range of the lens was $2.7-9 \mathrm{~mm}$, the viewing angle range was $101^{\circ}-30.4^{\circ}$, the shutter speed was $1 / 25 \mathrm{~s}$, the aperture was F1.6, and automatic white balance was not supported. The focal length of the wide-angle camera was adjusted to $2.7 \mathrm{~mm}$, the camera was focused manually to ensure that the image was clear, and the camera parameters remain unchanged during the test. The camera was installed on the top beam of greenhouse which is $3.75 \mathrm{~m}$ above the flora canopy, and the lens was angled straight downwards. Capture color image size is $1200 \times 1600 \times 3$, Canopy images of the greenhouse cucumbers were captured at times set from May 11, 2015 to June 29, 2015.

\subsection{Principle of image compression coding/decoding}

\section{for the floral canopy}

The floral canopy image can contain many arbitrarily shaped ROIs. To achieve nearly lossless compression of multiple regions of the floral canopy, we propose the method of ROI-MHC, which adopted the ROI-SPIHT coding for the image ROI and the EZW coding for the image BG. The idea was to achieve multiple local ROI priority coding under the situation of a fixed coding amount and to ensure that the ROI occupies a relatively high proportion of the coding amount while being able to reflect the global BG status.

Figure 1 shows the principle of floral canopy image coding/decoding. The demonstrated principle is as follows: firstly, automatic detection of the ROI is conducted according to the color features of the floral canopy image, and the ROI mask is generated and transformed to a ROI-discrete wavelet transform (DWT) mask in the wavelet domain; then, the original image is decomposed to the ROI and BG images (the proportion of ROI relative to the entire image is $a$, the proportion of $\mathrm{BG}$ relative to the entire image is $1-a$, and the range of $a$ is 0-1). The waveform transform is applied to the ROI and BG images to derive the wavelet coefficients, and the bit-plane of the ROI wavelet coefficient is lifted with respect to the ROI-DWT mask. SPIHT encoding is applied for the ROI to enable the priority coding of the ROI, whereas EZW coding is adopted for the BG; the reason is to acquire relatively many low-frequency coefficients while ensuring a relatively small proportion of coding, making the $\mathrm{BG}$ reconstructed image able to reflect the global status of the BG; finally, according to the compression ratio $B P P$ and the coding proportional coefficient $k$ ( $k$ is the proportion of ROI image coding, and $1-k$ is the proportion of $\mathrm{BG}$ image coding; $k$ is calculated from the ROI proportion $a$ and the weighing factor $r$ of the ROI coding amount, and the range of $k$ values is $0-1$ ), the coding of the ROI and BG are combined into the bit streams for information transmission or storage. The decoding of the image is the reverse process of coding, and this process can decode the reconstructed canopy image.

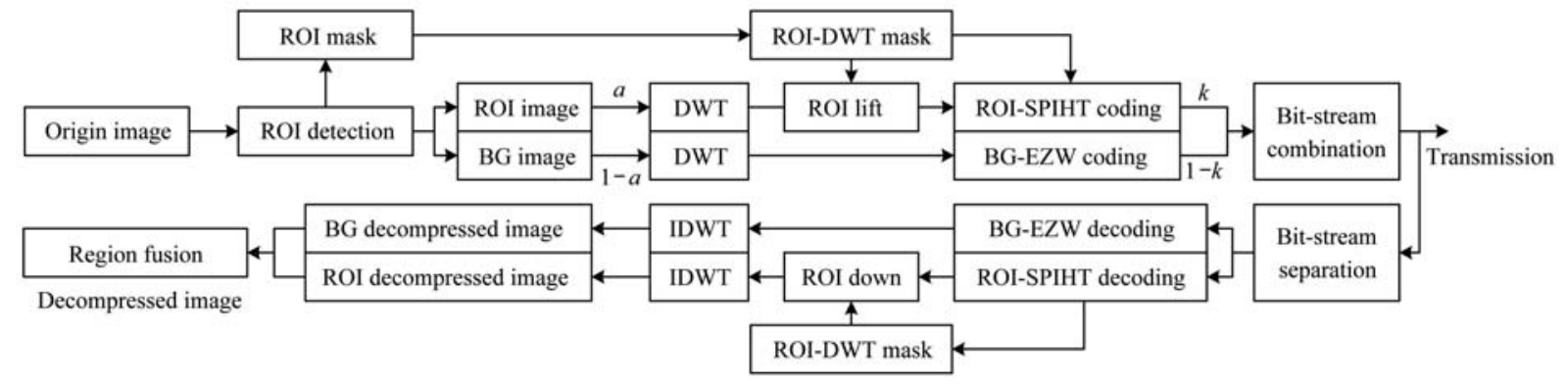

Figure 1 Diagram for the principle of coding/decoding for floral canopy image compression

\subsection{Identification of ROI mask for floral canopy} image

The ROI of the floral canopy image is primarily green. For the ROI identification of the floral canopy, the most widely applied methods at present include the ExG segmentation method, ExG-ExR method, 
normalized excess green $(\mathrm{Exg}=2 g-r-b)$ segmentation method, $\mathrm{H}$ colorimetric method of HSI color space, $\mathrm{Cr}$ chromatic aberration method of $\mathrm{YcbCr}$ color space, and $\mathrm{NDI}^{[6-9]}$. Sun et al. previously adopted the MExG-ExR method to carry out an in-depth study on the ROI identification of a floral canopy (a detailed analysis and discussion can be found in the literatures $[10,11]$ and are not included in this paper).

Figure $2 \mathrm{a}$ shows the original image of the floral canopy. Figure $2 b$ shows the region of ROI mask identification. (On the basis of previous reports ${ }^{[10,11]}$, to improve the accuracy of ROI identification, the connected domain was detected along with the processing of small region removal and gap filling inside the connected domain. Of course, to improve the efficiency of ROI identification, this post-processing flow could be omitted.) Figure 2c shows the mask information of the ROI mask in the three-layered wavelet domain, i.e., the ROI-DWT mask (the ROI region is expressed with 1 , and the BG region is expressed by 0 ), and Figures $2 \mathrm{~d}$ and $2 \mathrm{e}$ are the ROI and BG images, respectively.

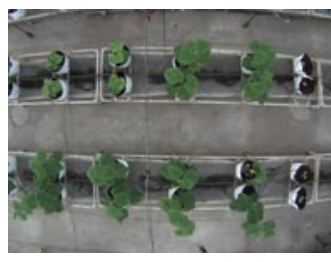

a. Original image

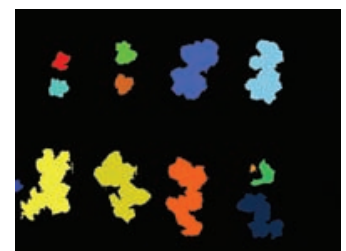

b. Mask

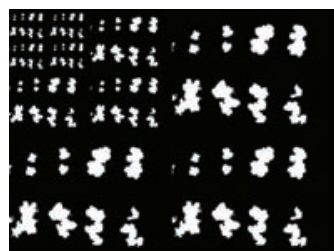

c. ROI-DWT mask

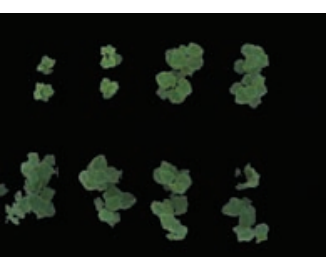

d. ROI

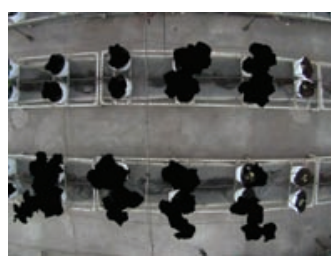

e. BG

Figure 2 Identification of ROI mask for floral canopy image

2.3 Algorithm of ROI bit-plane lifting and ROI-SPIHT coding

In this study, the ROI coding adopts the SPIHT algorithm that is a coding algorithm of an embedded bit-plane based on the number of layers. The wavelet coefficient of the high bit-plane will be priority coded. Therefore, to obtain the ROI coding priority, in this study the ROI wavelet coefficient was increased according to the ROI-DWT mask, i.e., the wavelet coefficient was shifted corresponding to the ROI mask in the wavelet domain by $\mathrm{S}$ bits, which is equivalent to multiplying the wavelet coefficient by $2 \mathrm{~S}$; for the SPIHT coding of the bit-plane, the ROI obtains priority coding ${ }^{[2,18]}$. Figure 3 shows the schematic diagram for the lifting of the ROI bit-plane, where $\mathrm{D}$ is the bit width of the wavelet coefficient amplitude, Sign is the bit-plane of the symbol, Mask is the bit-plane of the ROI mask, and $S$ is the lifting factor. When $S$ value is larger, the priority level of the $\mathrm{ROI}$ is higher relative to the $\mathrm{BG}$, and the image quality of the ROI is also better.

In this study, the ROI mask information was embedded into the standard SPIHT algorithm, and the bit streams contained the information of whether the wavelet coefficient belonged to the ROI. Upon decoding, we can move the bit-plane downward for the ROI wavelet coefficient to recover the image according to the ROI mask information ${ }^{[2,18]}$.

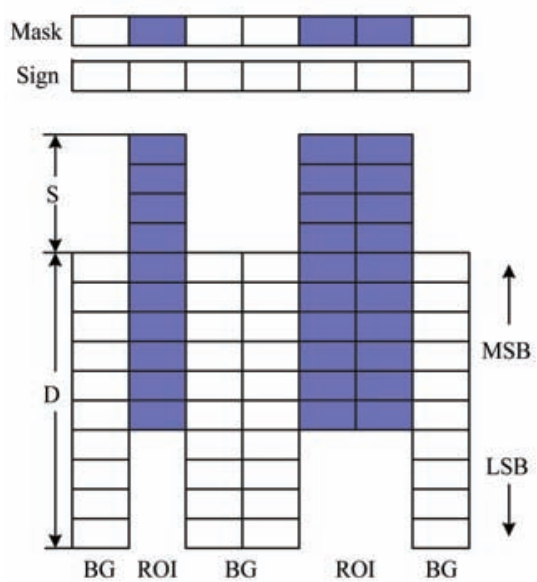

Figure 3 Schematic diagram for the lifting of the SPIHT spatial orientation tree.

As shown in Figure 4, the set of ROI-SPIHT coding algorithm procedures is defined as follows: $C(i, j)$ is the root node; $D(i, j)$ is the set of all descendant nodes of node $(i, j)$, but not including node $(i, j)$ itself; $O(i, j)$ is the set of direct descendant nodes corresponding to node $(i, j)$; $L(i, j)$ is the set of indirect descendant nodes corresponding to node $(i, j)$; and LIS is the linked list of insignificant sets, which is divided into category A: $D(i, j)$ and category B: $L(i, j)$. LIP is the linked list of insignificant nodes, and LSP is the linked list of significant nodes. 


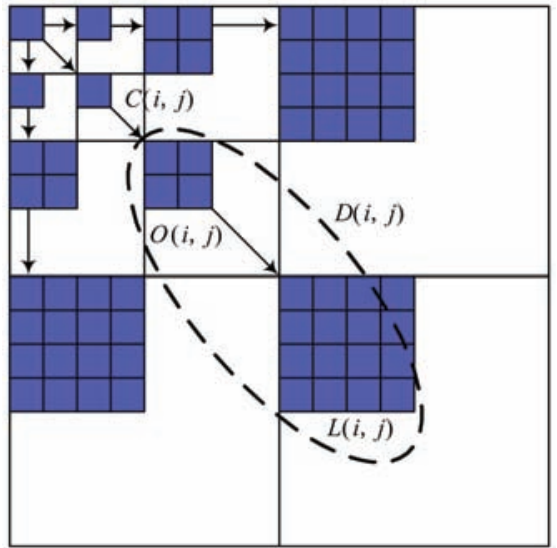

Figure 4 Schematic diagram for the structural relationship of the ROI bit-plane

The flow chart of ROI-SPIHT coding is as follows:

I) Initialization: the $n$-order wavelet decomposition of the original image was conducted, each individual self-zone was generated, and the wavelet coefficients were obtained; the initial door threshold $T_{0}$ was selected. For positive integer $n$, we have $n=\left[\log _{2}\left(\max _{(i, j)}\left\{\left|C_{i, j}\right|\right\}\right)\right]$, and LSP was set to the null set. All of the points $(i, j)$ in set $H$ were added into LIP, and the nodes $(i, j)$ with descendants in set $H$ were added into LIS.

II) Classification:

1) For every $(i, j)$ in LIS, output $S_{n}(i, j)$; if $S_{n}(i, j)=1$, move $(i, j)$ to LSP and output $\operatorname{Sgn}(i, j)$ and $R O I(i, j)$;

2) For every $(i, j)$ in LIS,

If node $(i, j)$ is of class A, output $\operatorname{Sn}(D(i, j))$;

If $\operatorname{Sn}(D(i, j))=1$, for every $(k, l)$ of $O(i, j)$, Output $\operatorname{Sn}(k, l)$.

If $\operatorname{Sn}(k, l)=1$, add $(k, l)$ to LSP and output $\operatorname{Sgn}(k, l)$ and $R O I(k, l)$; otherwise, add $(k, l)$ to the end of LIP.

If $L(i, j)$ is not null, add $(i, j)$ as class B to the end of LIS; otherwise, delete $(i, j)$.

If node $(i, j)$ is of class B, output $\operatorname{Sn}(L(i, j))$;

If $\operatorname{Sn}(L(i, j))=1$, add all nodes $O(i, j)$ to LIS, set them as class A, and delete $(i, j)$.

III) Refine: for LSP, except for node $(i, j)$ generated at the current iteration, output the $n^{\text {th }}$ node of $|c(i, j)|$.

IV) Update the quantification order; this procedure gradually decreases $n$ by 1 .

The difference between the ROI-SPIHT algorithm and standard SPIHT is that when node $(i, j)$ is added to LSP every time, after outputting $\operatorname{Sgn}(i, j)$, the information of one bit for $\operatorname{ROI}(i, j)$ is appended. In the coding process, the none-zero coefficients all contain ROI mask information, whereas the 0 coefficients do not affect the image recovery whether they belong to ROI coefficients or not. Therefore, the bit streams only contain the ROI information of important coefficients, and the coding of ROI information also has the embedded, truncatable property.

\section{Results and discussion}

\subsection{Evaluation index of plant image compression quality}

To objectively evaluate the quality of plant image compression, in this study four image evaluation indexes were selected, peak signal-to-noise ratio (PSNR), mean square error $(M S E)$, similarity degree $(X S D)$ and texture distortion $(T D)^{[2,20]}$; the calculation formulas are shown as Equations (1)-(4). The compression and reconstruction qualities were statistically analyzed for the ROI, BG, and ALL (the composite image of ROI and BG) under the condition of different compression rates (in this study, the compression rate is expressed as the bit rate).

$$
\begin{gathered}
P S N R=10 \lg \left(\frac{255^{2}}{M S E}\right) \\
M S E=\frac{1}{3 M N} \sum_{x=0}^{M-1} \sum_{y=0}^{N-1} \sum_{z=1}^{3}[\hat{f}(x, y, z)-f(x, y, z)]^{2}
\end{gathered}
$$

$X S D=$

$$
\begin{gathered}
\frac{\sum_{x=0}^{M} \sum_{y=0}^{N} \sum_{z=1}^{3}\left(f(x, y, z)-f_{\text {avg }}(x, y, z)\right)\left(\hat{f}(x, y, z)-\hat{f}_{\text {ayg }}(x, y, z)\right)}{\sqrt{\left(\sum_{x=0}^{M} \sum_{y=0}^{N} \sum_{z=1}^{3}\left(f(x, y, z)-f_{\text {avg }}(x, y, z)\right)\right)^{2}\left(\sum_{x=0}^{M} \sum_{y=0}^{N} \sum_{z=1}^{3}\left(\hat{f}(x, y, z)-\hat{f}_{\text {avg }}(x, y, z)\right)\right)^{2}}} \\
T D=\frac{2 A_{f} C_{f} A_{\hat{f}} C_{\hat{f}}}{\left(A_{f} C_{f}\right)^{2}+\left(A_{\hat{f}} C_{\hat{f}}\right)^{2}}
\end{gathered}
$$

where, $f(x, y, z)$ represents the original image; $\hat{f}(x, y, z)$ represents the reconstructed image; $M$ is the total number of rows in the image; $N$ is the total number of columns in the image; $(x, y)$ are the coordinates of the image; $z=1$ is channel $R$ of the image; $z=2$ is channel $G$ of the image; $z=3$ is channel $B$ of the image; $f_{\text {avg }}(x, y, z)$ represents the average gray value of the original image; $\hat{f}_{\text {avg }}(x, y, z)$ represents the average gray value of the reconstructed image; $A_{f}$ and $C_{f}$ are the angular second moment and contrast of the original image, respectively; and $A_{\hat{f}}$ and 
$C_{\hat{f}}$ are the angular second moment and contrast of the reconstructed image, respectively.

\subsection{Parameter setting of image compression}

Prior to the coding of image compression, it needs to set reasonable parameters for compression, which mainly include the compression rate $B P P$, lifting factor $S$ of the ROI bit-plane, and the weighing factor $r$ of the ROI coding amount. The compression rate BPP is determined according to the actual coding amount (the original color image uses 24 bits to express one pixel, and $B P P$ is the number of bits for one pixel after image compression; the range for the value of the bit-plane lifting factor $S$ is $0<S<\mathrm{D}$ (bit width of wavelet coefficient)). The condition $S=0$ indicates a lack of ROI enhancement. The proportional coefficient of ROI priority coding amount, $k$, is affected by the weighing factor $r$ of the ROI coding amount and the proportion of the ROI relative to the entire image, $a$ (the floral canopy region of cucumber changes continuously during the growing period, and therefore, $a$ is a variable), and the relationship of $k, r$ and $a$ satisfies Equation (5). As shown in Figure 5, the condition $r=1$ indicates that the weight of ROI and BG coding is the same; the condition $r>1$ indicates that ROI requires more coding per unit area than BG to express the information of these pixels. The larger the $r$ value is, the larger the coding amount of unit ROI pixel, whereas that of BG is less. For instance, assuming that the total coding amount is $1000 \mathrm{~kb}$ and $a=0.2$, when $r=2$, ROI occupies a coding amount of $333 \mathrm{~kb}$, and BG occupies a coding amount of $667 \mathrm{~kb}$; when $r=4$, ROI occupies a coding amount of $500 \mathrm{~kb}$, and BG occupies a coding amount of $500 \mathrm{~kb}$. We can write

$$
k=\frac{r \alpha}{1-\alpha+r a}
$$

where, $k$ is the proportional coefficient of the ROI priority coding amount; $a$ is the proportion of the ROI relative to the entire image; and $r$ is the weighing factor of the ROI coding amount.

According to the aforementioned principle of image compression coding/decoding, we selected a canopy image of cucumber population taken on June 12, 2015; the size of the colorful image is $1200 \times 1600 \times 3$. In particular, the proportion of the ROI and $\mathrm{BG}$ relative to

the entire image is 0.18 and 0.82 , respectively (i.e., $a=0.18$ ). During coding, the ROI has coding priority $(S=4)$ through the improvement of the bit-plane, BPP is $1.2 \mathrm{bit} / \mathrm{pixel}$ (bpp), and the range of $r$ is $0-20$. Figure 6 shows the curve for the variation in the PSNR of the ROI and $\mathrm{BG}$ reconstructed images with the $r$ value. The larger the $r$ value, the larger the PSNR of the ROI reconstructed image. The quality of ROI compression is improved, whereas the PSNR of the BG reconstructed image becomes smaller, which indicates that the quality of $\mathrm{BG}$ compression decreases.

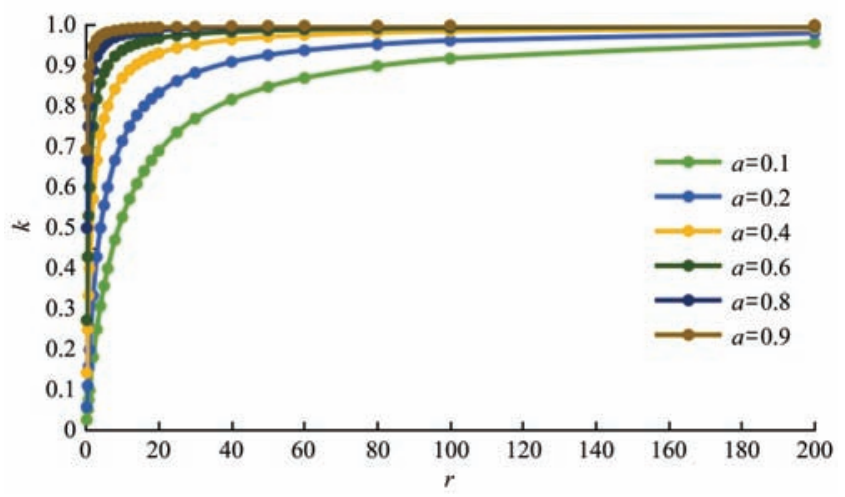

Figure 5 Relationship between $r, a$ and $k$

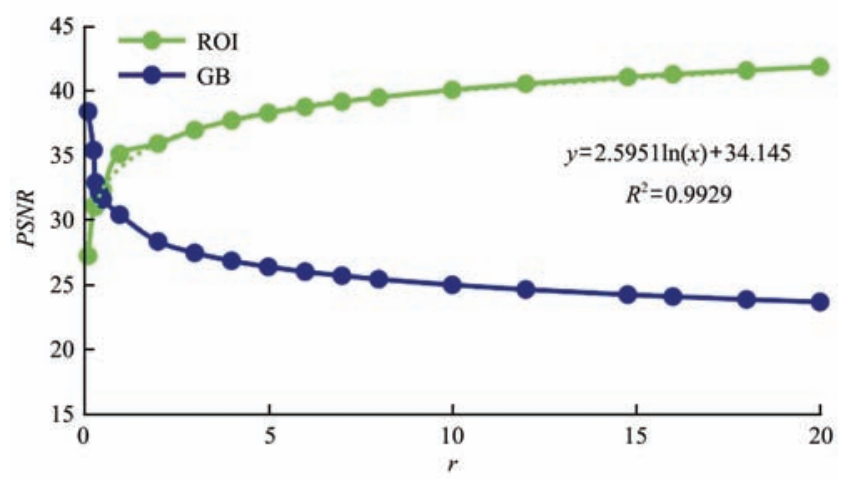

Figure 6 Influence of $r$ on image compression quality (1.2 bpp)

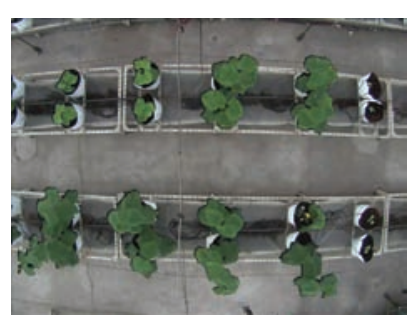

$r=0.1(1.2 \mathrm{bpp})$

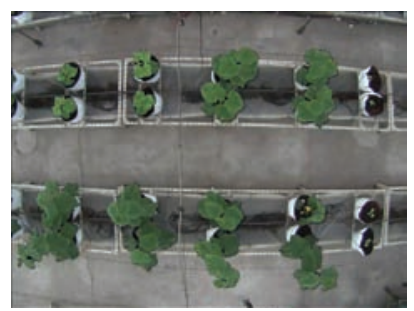

$r=5(1.2 \mathrm{bpp})$

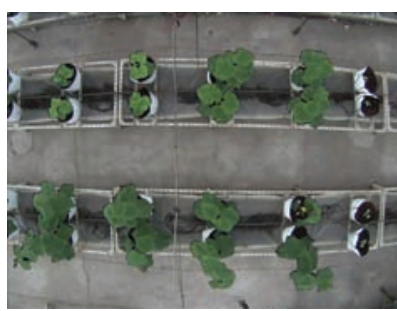

$r=0.5(1.2 \mathrm{bpp})$

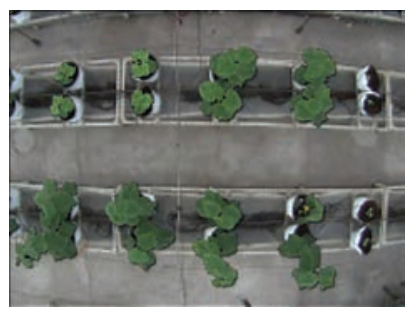

$r=10(1.2 \mathrm{bpp})$

Figure 7 Influence of $r$ on reconstruction quality of ROI and BG 
Figure 7 shows the reconstructed composite image for $r=0.1, r=0.5, r=5$, and $r=10$. From the image, it can be observed that when $r<1$, the ROI is blurrier than the BG; when $r>1$, the ROI is clear, whereas the BG is blurry. In subsequent section 3.3, the quality of compression and reconstruction were quantitatively evaluated.

\subsection{Analysis of image compression quality}

In this study, the indexes of PSNR, MSE, XSD, and $T D$ were adopted for image evaluation. For various compression rates of 0.07-1.09 bpp, $r=4$ was selected after repeated experiments ${ }^{[2]}$. The quality of image reconstruction was statistically analyzed for the ROI, BG, and ALL when adopting the image compression algorithm of ROI-MHC, and compared with the standard SPIHT coding/decoding.

As shown in Figure 8a, the PSNR of the ROI reconstructed image is clearly higher than that of the $\mathrm{BG}$ and ALL. Although the PSNR of the reconstructed image using standard SPIHT coding/decoding is higher than ALL, the image reconstruction quality of the ROI is

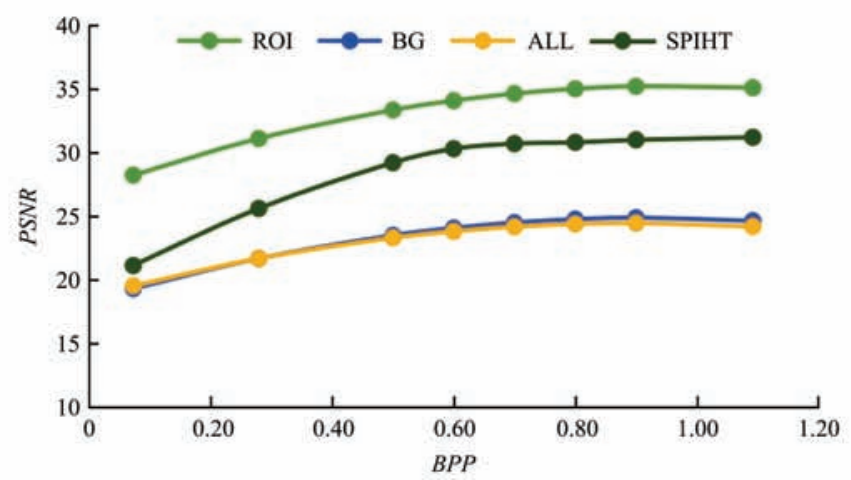

a. Peak signal-to-noise ratio

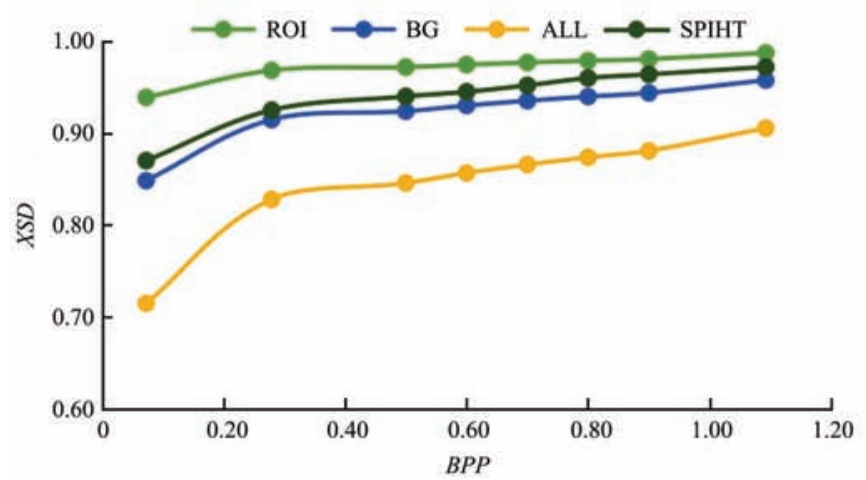

c. Similarity degree guaranteed. The PSNR of the ROI is $42.65 \%$ higher on average than the $P S N R$ of the BG, $43.95 \%$ higher on average than the PSNR of ALL, and $16.84 \%$ higher than the PSNR of the SPIHT reconstructed image, which indicates that ROI-MHC can improve the quality of ROI image reconstruction and that the performance is superior to standard SPIHT coding/decoding.

Figure $8 \mathrm{~b}$ shows the MSE curve of the ROI-MHC reconstructed image and the original image; the mean MSE of the ROI, BG, and ALL relative to the corresponding region of the original image is 44.24 , 391.53, and 352.08, respectively. The mean MSE between the SPIHT reconstructed image and the original image is 129.24 , which indicates that the MSE of the ROI reconstructed image is the smallest. The reconstruction error of the gray value for different channels of the ROI-MHC reconstructed image is smaller than that of the SPIHT reconstructed image, and the MSE is smaller than that of the SPIHT reconstruction image by $50.17 \%$.

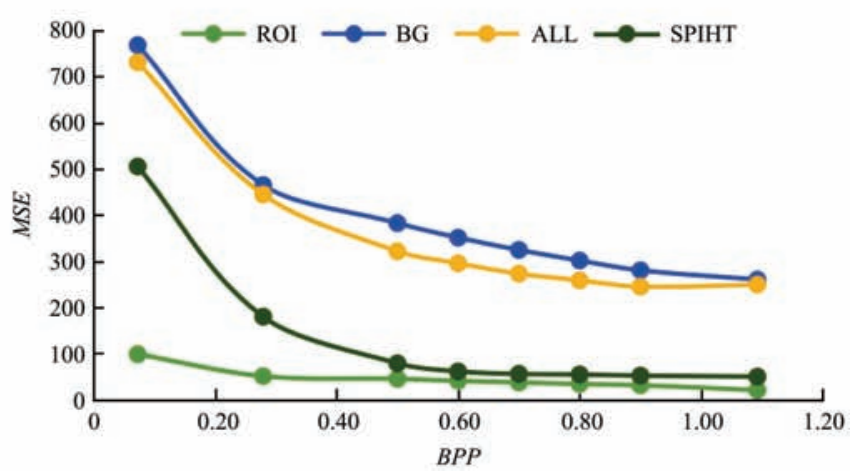

b. Mean square error

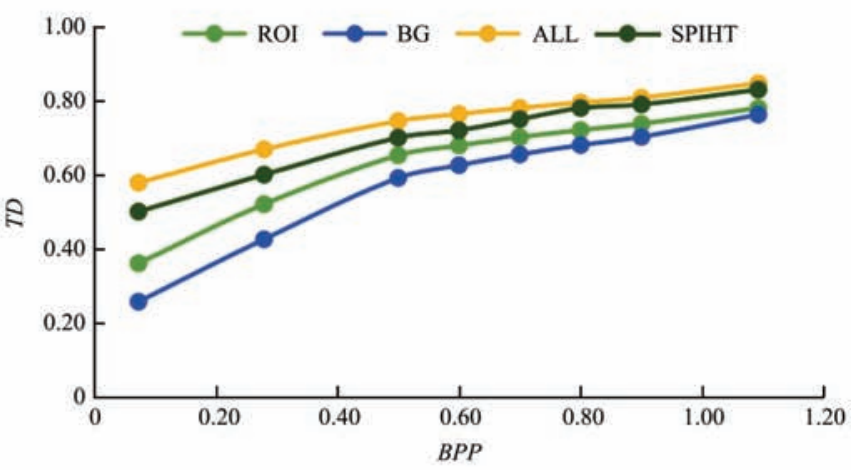

d. Texture distortion

Figure 8 Image compression performance under different compression rates

Figure 8c shows the curve of the XSD for ROI-MHC reconstructed image and original image. The average $X S D$ of the ROI, BG, and ALL relative to the corresponding region of the original image are 0.972 , 0.924 , and 0.846 , respectively, and the average XSD between the SPIHT reconstructed image and the original 
image is 0.941 , which indicates that the $X S D$ of the ROI reconstructed image is the highest. The reconstruction performance of the ROI-MHC is better than the SPIHT, but, the coding amount of the BG image is less than ROI, which leads to the decrease of the XSD of BG and ALL image.

Figure $8 \mathrm{~d}$ shows the curve of the $T D$ for ROI-MHC reconstructed image and original image. The average $T D$ of the ROI, BG, and ALL relative to the corresponding region of the original image are 0.643 , 0.587 , and 0.748 , respectively, and the average $T D$ between the SPIHT reconstructed image and the original image is 0.709 , which indicates that the $T D$ of the $\mathrm{BG}$ is the most severe and that the TD of ALL is the smallest. This result occurs because the difference between the gray values of the ROI and BG is significant and because ALL includes the marginal area of the ROI and BG.

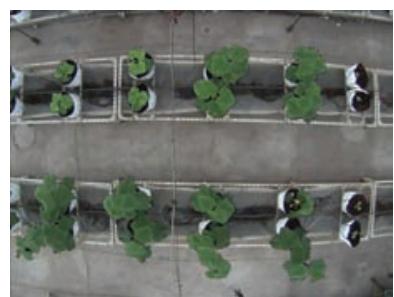

ROI-MHC: 0.96 bpp

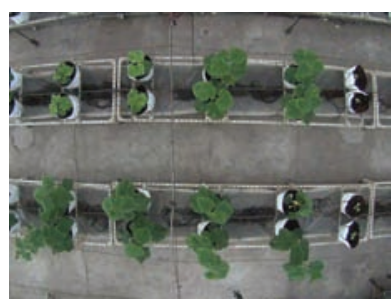

SPIHT: $0.96 \mathrm{bpp}$

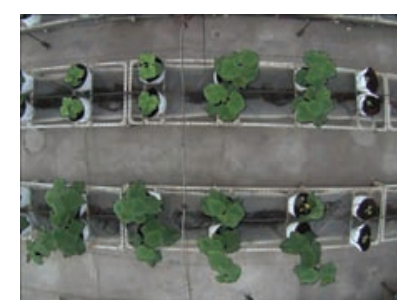

ROI-MHC: $0.56 \mathrm{bpp}$

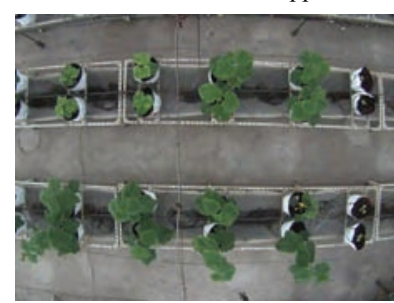

SPIHT: $0.56 \mathrm{bpp}$

Therefore, the texture features of ALL (i.e., the ROI and $\mathrm{BG}$ reconstructed composite image) is the most similar to the texture of the original image. The $T D$ of the ROI is smaller than the $T D$ of the $\mathrm{BG}$ and is $10.00 \%$, higher than the TD of the SPIHT reconstructed image. However, the $T D$ of the ROI-MHC reconstructed image ALL is $6.24 \%$ smaller than the $T D$ of the SPIHT reconstructed image.

Figure 9 shows the reconstruction results of the ROI-MHC and SPIHT for $0.96 \mathrm{bpp}, 0.56 \mathrm{bpp}, 0.28 \mathrm{bpp}$ and $0.1 \mathrm{bpp}$, respectively. The results indicated that the ROI-MHC image compression algorithm proposed in this paper can ensure that the ROI reconstruction quality is higher than the $\mathrm{BG}$ reconstruction quality. As the compression rate increases, the quality of ROI reconstruction is relatively high, whereas the reconstruction quality of SPIHT coding/decoding continuously declines.

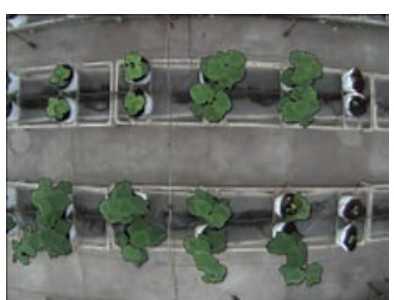

ROI-MHC: 0.28 bpp

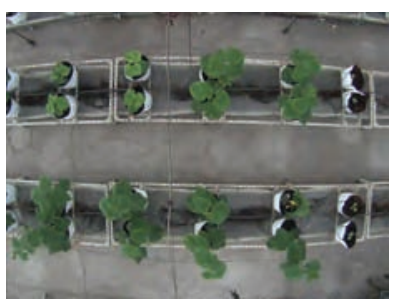

SPIHT: $0.28 \mathrm{bpp}$

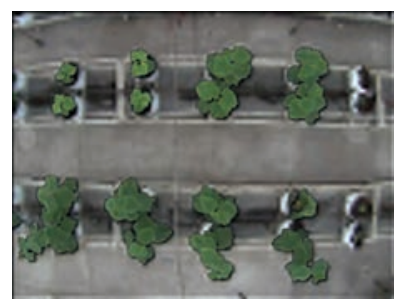

ROI-MHC: $0.1 \mathrm{bpp}$

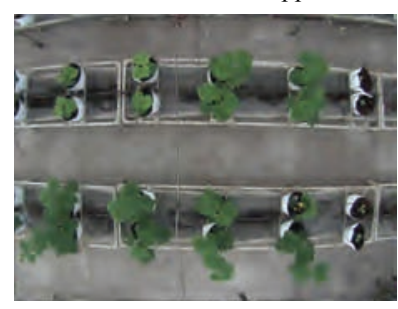

SPIHT: $0.1 \mathrm{bpp}$

Figure 9 Reconstruction plot of ROI-MHC and SPIHT with different bit rates

\subsection{Applicability analysis of image compression algorithm}

To analyze the applicability of the ROI-MHC image compression algorithm proposed in this study, the images were collected during the experimental period. The proportions of the ROI relative to the image, $a$, are 0.12 , $0.27,0.45$, and 0.69 , and the compression rates are 0.08 bpp, $0.28 \mathrm{bpp}, 1.00 \mathrm{bpp}$ and $2.00 \mathrm{bpp}$. For the weighing factor $r=4$, a statistical analysis was conducted on the indexes of PSNR, MSE, XSD, and TD for the reconstruction quality of image compression. The image reconstructed by ROI-MHC image compression is shown in Figure 10.
As shown in Table 1, the PSNR of the ROI for the ROI-MHC compressed and reconstructed image is higher than that of the BG by $32.51 \%$ on average, higher than that of ALL by $48.94 \%$ on average, and higher than that of the SPIHT reconstructed image by $10.97 \%$ on average, which indicates that the reconstruction quality of the ROI image is the highest. Compared to corresponding regions of the original image, the average $M S E$ of the ROI, BG, ALL, and reconstructed image of SPIHT coding/decoding are 117.75, 482.98, 741.08, and 147.53, respectively, which indicates that the $M S E$ of the ROI reconstructed image is the smallest, which is smaller than the standard SPIHT algorithm. 


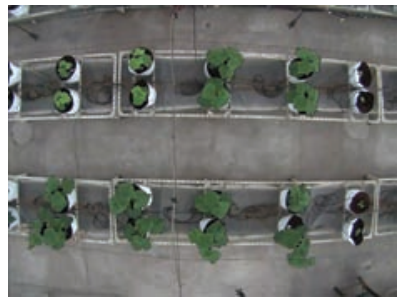

$a=0.12(2.00 \mathrm{bpp})$

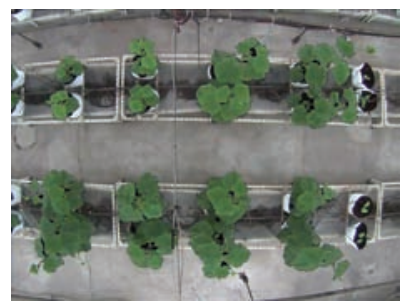

$a=0.27(2.00 \mathrm{bpp})$

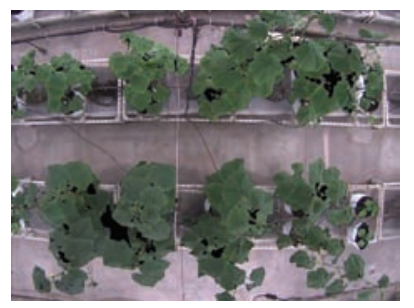

$a=0.45(2.00 \mathrm{bpp})$

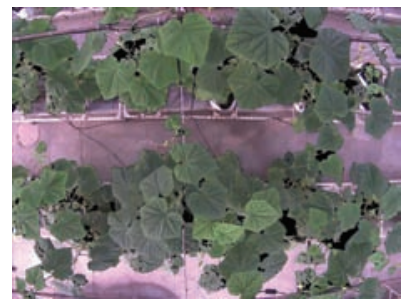

$a=0.69$ (2.00 bpp)

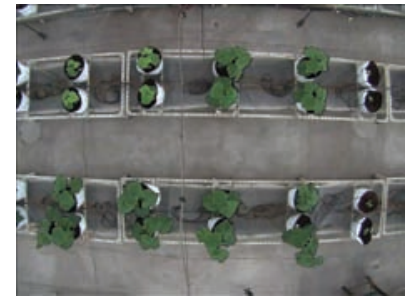

$a=0.12(1.00 \mathrm{bpp})$

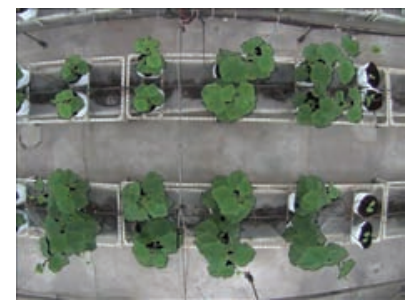

$a=0.27$ (1.00 bpp)

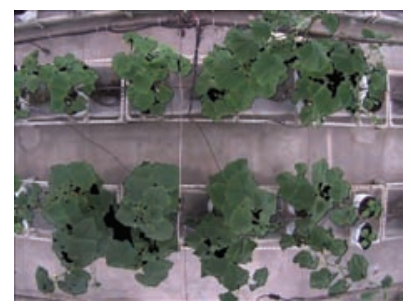

$a=0.45$ (1.00 bpp)

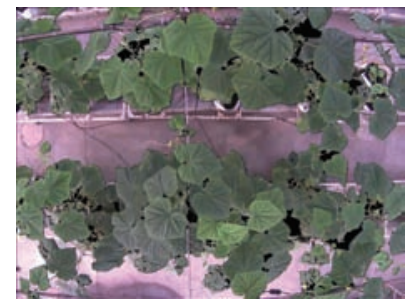

$a=0.69(1.00 \mathrm{bpp})$

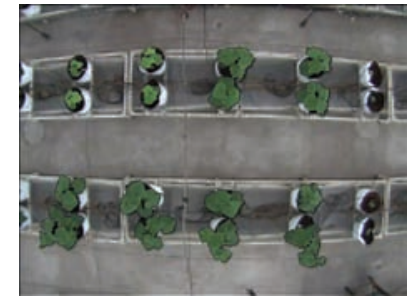

$a=0.12(0.28 \mathrm{bpp})$

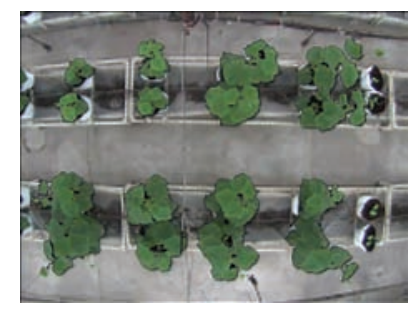

$a=0.27$ (0.28 bpp)

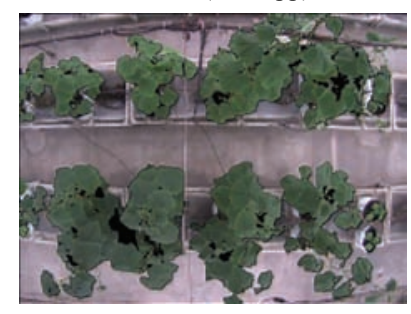

$a=0.45$ (0.28 bpp)

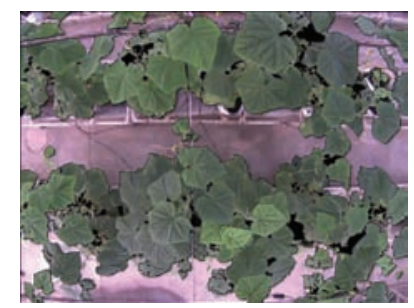

$a=0.69(0.28 \mathrm{bpp})$

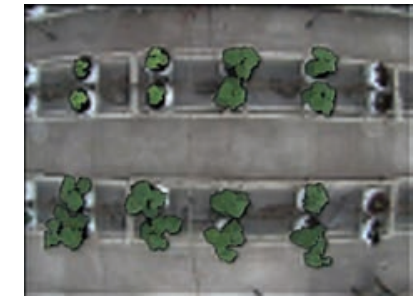

$a=0.12(0.08 \mathrm{bpp})$

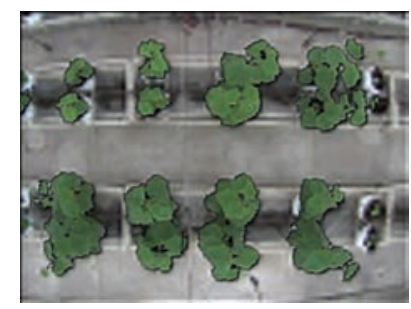

$a=0.27$ (0.08 bpp)

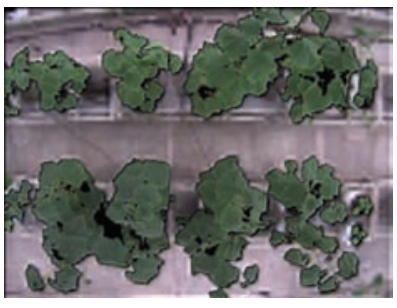

$a=0.45$ (0.08 bpp)

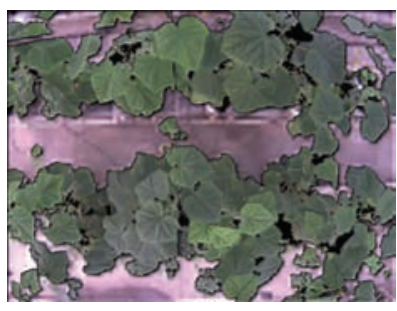

$a=0.69$ (0.08 bpp)

Figure 10 Reconstructed image by ROI-MHC image compression

Table 1 Compression performance by the ROI-MHC

\begin{tabular}{|c|c|c|c|c|c|c|c|c|c|c|c|c|c|c|c|c|c|}
\hline \multirow{2}{*}{$a$} & \multirow{2}{*}{$P P$} & \multicolumn{4}{|c|}{$P S N R$} & \multicolumn{4}{|c|}{ MSE } & \multicolumn{4}{|c|}{$X S D$} & \multicolumn{4}{|c|}{$T D$} \\
\hline & & ROI & BG & ALL & SPIHT & ROI & BG & ALL & SPIHT & ROI & BG & ALL & SPIHT & ROI & BG & ALL & SPIHT \\
\hline \multirow{5}{*}{0.12} & 0.08 & 29.31 & 19.38 & 19.51 & 24.69 & 76.31 & 749.19 & 728.57 & 220.87 & 0.93 & 0.82 & 0.70 & 0.91 & 0.51 & 0.48 & 0.65 & 0.58 \\
\hline & 0.28 & 32.25 & 21.68 & 21.62 & 27.92 & 38.74 & 441.53 & 448.15 & 105.01 & 0.96 & 0.90 & 0.82 & 0.93 & 0.69 & 0.62 & 0.76 & 0.72 \\
\hline & 1.00 & 36.36 & 24.58 & 24.18 & 28.78 & 15.05 & 226.39 & 248.32 & 68.33 & 0.99 & 0.95 & 0.90 & 0.98 & 0.76 & 0.73 & 0.84 & 0.81 \\
\hline & 2.00 & 42.00 & 30.40 & 28.27 & 30.76 & 7.65 & 59.31 & 96.83 & 54.58 & 1.00 & 0.99 & 0.96 & 0.99 & 0.94 & 0.88 & 0.99 & 0.97 \\
\hline & AVG & 34.98 & 24.01 & 23.39 & 28.04 & 34.44 & 369.11 & 380.47 & 112.20 & 0.97 & 0.91 & 0.85 & 0.95 & 0.73 & 0.68 & 0.81 & 0.77 \\
\hline \multirow{5}{*}{0.27} & 0.08 & 25.87 & 18.23 & 18.28 & 23.99 & 168.16 & 976.57 & 966.84 & 259.18 & 0.94 & 0.88 & 0.72 & 0.92 & 0.47 & 0.39 & 0.67 & 0.48 \\
\hline & 0.28 & 28.65 & 20.71 & 20.26 & 26.31 & 88.67 & 551.77 & 612.77 & 151.93 & 0.97 & 0.93 & 0.82 & 0.96 & 0.64 & 0.56 & 0.75 & 0.81 \\
\hline & 1.00 & 32.46 & 23.83 & 22.37 & 29.48 & 36.87 & 269.04 & 376.84 & 73.24 & 0.99 & 0.97 & 0.89 & 0.98 & 0.78 & 0.79 & 0.90 & 0.93 \\
\hline & 2.00 & 38.00 & 29.63 & 24.55 & 30.45 & 14.33 & 70.87 & 228.07 & 58.62 & 1.00 & 0.99 & 0.94 & 0.99 & 0.93 & 0.97 & 1.00 & 0.95 \\
\hline & AVG & 31.25 & 23.10 & 21.36 & 27.56 & 77.01 & 467.06 & 546.13 & 135.74 & 0.97 & 0.94 & 0.84 & 0.96 & 0.71 & 0.68 & 0.83 & 0.79 \\
\hline \multirow{5}{*}{0.45} & 0.08 & 23.94 & 17.57 & 17.10 & 23.17 & 262.48 & 1138.40 & 1267.90 & 313.53 & 0.92 & 0.88 & 0.68 & 0.91 & 0.40 & 0.31 & 0.87 & 0.42 \\
\hline & 0.28 & 26.62 & 20.14 & 18.56 & 25.31 & 141.58 & 630.15 & 905.46 & 191.28 & 0.96 & 0.94 & 0.77 & 0.95 & 0.58 & 0.46 & 0.91 & 0.67 \\
\hline & 1.00 & 30.30 & 23.40 & 19.94 & 29.49 & 60.65 & 296.98 & 659.42 & 73.02 & 0.98 & 0.97 & 0.83 & 0.98 & 0.79 & 0.70 & 0.99 & 0.96 \\
\hline & 2.00 & 36.00 & 29.02 & 20.91 & 30.12 & 30.46 & 81.43 & 527.50 & 63.25 & 1.00 & 0.99 & 0.87 & 0.99 & 0.89 & 0.93 & 0.90 & 0.98 \\
\hline & AVG & 29.22 & 22.53 & 19.13 & 27.02 & 123.79 & 536.74 & 840.07 & 160.27 & 0.96 & 0.95 & 0.79 & 0.96 & 0.67 & 0.60 & 0.92 & 0.76 \\
\hline \multirow{5}{*}{0.69} & 0.08 & 21.06 & 17.25 & 15.97 & 22.95 & 509.67 & 1226.10 & 1645.00 & 329.33 & 0.90 & 0.89 & 0.60 & 0.91 & 0.38 & 0.25 & 0.92 & 0.49 \\
\hline & 0.28 & 23.72 & 20.04 & 17.18 & 24.90 & 276.22 & 643.57 & 1244.50 & 210.35 & 0.94 & 0.94 & 0.70 & 0.94 & 0.42 & 0.38 & 0.94 & 0.73 \\
\hline & 1.00 & 27.56 & 23.58 & 18.18 & 27.82 & 113.96 & 285.36 & 989.56 & 107.40 & 0.98 & 0.98 & 0.76 & 0.97 & 0.73 & 0.61 & 0.98 & 0.95 \\
\hline & 2.00 & 32.00 & 29.04 & 18.53 & 29.07 & 43.33 & 81.04 & 911.60 & 80.55 & 1.00 & 0.99 & 0.79 & 0.99 & 0.92 & 0.89 & 1.00 & 0.96 \\
\hline & AVG & 26.08 & 22.48 & 17.46 & 26.19 & 235.79 & 559.02 & 1197.66 & 181.91 & 0.95 & 0.95 & 0.71 & 0.95 & 0.61 & 0.53 & 0.96 & 0.78 \\
\hline
\end{tabular}


Compared to corresponding regions of the original image, the average $X S D$ between the ROI, BG, ALL, and SPIHT reconstructed image are $0.96,0.94,0.80$, and 0.95 , respectively, which indicates that the error of gray value for different channels of the ROI reconstructed image is the smallest. Compared to corresponding regions of the original image, the average $T D$ of the ROI, BG, ALL, and SPIHT reconstructed image are $0.68,0.62,0.87$ and 0.78 , respectively, which indicates that the $T D$ of the ALL reconstructed image using ROI-MHC is the smallest. Although the TD of the ROI and BG is larger than that of SPIHT, the texture features of the plant image are mainly manifested on the boundary between the ROI and BG, and therefore, it is normal that the $T D$ of the ROI and $\mathrm{BG}$ is larger than that of ALL and SPIHT. The TD of the reconstructed composite image using ROI-MHC is smaller than that of SPIHT, and consequently, ROI-MHC achieves the effect of high-quality reconstruction.

According to Table 1, it can be observed that as $a$ increases, the quality of ROI-MHC compression and reconstruction exhibits a gradual declining trend. The major reason is that the gradual increase of ROI proportion requires more wavelet coefficients to express the ROI, whereas the analysis in section 3.4 adopts a fixed weighing factor $(r=4)$. Therefore, as $a$ increases, the reconstruction quality of ROI-MHC declines. In section 3.2, the influence of $r$ on the image reconstruction quality was discussed. The larger the $r$ value is, the higher the ROI reconstruction quality and the lower the BG reconstruction quality. Therefore, when $a$ increases, $r$ can be appropriately adjusted to achieve high-quality image reconstruction of ROI-MHC.

The quality of the ROI-MHC image compression algorithm were affected by $B P P, a$, and $r$. In order to ensure the reconstruction quality of ROI, according to test data statistics, to establish the regression function of $P S N R$ by the variables: $B P P, a$ and $r$ (PSNR= $\mathrm{A} \times B P P+\mathrm{B} \times a+\mathrm{C} \times r+\mathrm{D}, \mathrm{A}, \mathrm{B}, \mathrm{C}$ and $\mathrm{D}$ were the regression coefficients, were 5.879, $-14.718,0.555$ and 28.797, respectively, in this study, $R^{2}$ was $0.9111, S E$ was 1.751 , $B P P$ was ranged from 0.08 to $2.00, a$ was ranged from 0.12 to $0.69, r$ was ranged from 1.00 to 20.00 ), $B P P$ was set by the amount of bandwidth, storage requirements, and the reconstruction quality, $a$ was the proportion of the canopy area in the image, so $r$ value can be dynamically calculated under the different $P S N R$ condition by artificial setting. At the same time, it is possible to set the $r$ fragment function according to the $P S N R, B P P$ and $a$, to achieve high quality ROI image reconstruction.

\section{Conclusions}

In this study, an image compression algorithm of a floral canopy was proposed based on ROI-MHC, and the main conclusions were obtained as follows:

(1) On the allocation method of the coding amount for the ROI and BG, we proposed the weighing factor $r$ and constructed the relationship between the proportion of the ROI coding amount $(k)$, weighing factor $(r)$, and proportion of ROI pixels relative to the total image $(a)$, achieving dynamic adjustment for the proportion of the ROI and $\mathrm{BG}$ coding amount.

(2) We adopt the four image evaluation indexes $P S N R$, $M S E, X S D$, and $T D$ to objectively and quantitatively evaluate the performance of the image compression algorithm. The results indicate that in the range of image compression rate from $0.07 \mathrm{bpp}$ to $1.09 \mathrm{bpp}(r=4)$, the PSNR of the ROI reconstructed image is $42.65 \%$ higher on average than that of the BG, $43.95 \%$ higher on average than that of the ROI-MHC reconstructed image, and $16.84 \%$ higher on average than that of the SPIHT reconstructed image, suggesting that the ROI-MHC image compression algorithm can improve the quality of ROI image reconstruction and that its performance is superior to the standard SPIHT algorithm.

(3) The results of applicability analysis for the ROI-MHC image compression algorithm indicate that the quality of the ROI reconstructed image is the best and that its performance is better than standard SPIHT. Moreover, when the weighing factor $r$ is a fixed value, as the proportion of the ROI, $a$, increases, the quality of ROI image reconstruction will gradually decline. Therefore, in the application of the ROI-MHC image compression algorithm, we can dynamically configure $r$ according to $a$ to achieve high-quality reconstruction of the ROI image.

In this study, ROI automatic detection is conducted according to the color features of the floral canopy, the 
original image is decomposed to the ROI and BG images, to realize priority coding of the multiple local arbitrary shape ROI by ROI-SPIHT coding, and EZW coding is adopted for the $\mathrm{BG}$, and according to the coding proportion, generated embedded codestream. At the same rate, ROI-MHC image compression quality is higher than the current similar compression algorithm, to achieve high quality ROI image reconstruction, has a good application prospects.

\section{Acknowledgements}

We acknowledge that this work was financial support provided by the Natural Science Fundation of Jiangsu Province: Youth Fund (Grant No. BK20170727), the Fundamental Research Funds for the Central Universities (Grant No. KYGX201703), the Natural Science Fundation of Jiangsu Province: Youth Fund (Grant No. BK20150686). The authors also thank the editor and anonymous reviewers for providing helpful suggestions for improving the quality of this manuscript.

\section{[References]}

[1] Sun G X, Li Y B, Zhang Y, Wang X C, Chen M, Li X, et al. Nondestructive measurement method for greenhouse cucumber parameters based on machine vision. Engineering in Agriculture, Environment and Food, 2016; 9(1): 70-78

[2] Xu Y, Xu Z Y, Zhang Q H, Zuo H R. A mask embedded spiht algorithm for arbitrary shape ROI coding. Opto-Electronic Engineering, 2009; 36(9): 118-124.

[3] Xu Y, Xu Z Y, Zhang Q H. Arbitrary shaped ROI image coding using Run-length coding and generalized Exp-Golomb coding. Opt. Precision Eng., 2011; 19(1): 175-181.

[4] Wang Y Y, Huang D Q. Compression for UAV reconnaissance images. Opt. Precision Eng., 2014; 22(5): $1363-1370$

[5] Zuo Z Y, Lan X, Deng L H, Yao S K, Wang X P. An improved medical image compression technique with lossless region of interest. Optik, 2015; 126: 2825-2831.

[6] Perez-Jimenez A, Lopez F, Benlloch J V, Christensen S. Color and shape analysis techniques for weed detection in cereal fields. Computer and Electronics in Agriculture, 2000; 25(3): 197-212.
[7] Hunt E R, Cavigelli M, Daughtry C T, Mcmurtrey J, Walthall S L. Evaluation of digital photography from model aircraft for remote sensing of crop biomass. Precision Agriculture, 2005; 6(4): 359-378.

[8] Meyer G E, João C N. Verification of color vegetation indices for automated crop imaging applications. Computer and Electronics in Agriculture, 2008; 63(2): 282-293.

[9] Meyer G E, João C N, David D J, Timothy W H. Intensified fuzzy clusters for classifying plant, soil, and residue regions of interest from color images. Computer and Electronics in Agriculture, 2004; 42(3): 161-180.

[10] Sun G X, Li Y B, Wang X C, Hu G Y, Wang X, Zhang Y. Image segmentation algorithm for greenhouse cucumber canopy under various natural lighting conditions. Int $\mathbf{J}$ Agric \& Biol Eng, 2016; 9(3): 130-138.

[11] Sun G X, Wang X C, Yan T T, Li X, Chen M, Shi Y Y. Inversion method of flora growth parameters based on machine vision. Transactions of the CSAE, 2014; 30(20): 187-195. (in Chinese)

[12] Hao H W, Jiang R R, Shi Y S. An image compression algorithm for circle shaped ROI. Acta Automatica Sinica, 2008; 34(5): 601-604.

[13] Kumarayapa A, Zhang Y. More efficient ground truth ROI image coding technique: implementation and wavelet based application analysis. Journal of Zhejiang University: Science A (S1673-565X), 2007; 8(6): 835-840.

[14] Taubman D S, Marcellin M W. JPEG 2000 image compression fundamentals, standards and practice. Beijing: Publishing House of Electronics Industry, 2004.

[15] Zhang L B, Yu X C. Multiple region of interest image coding based on classification bitplane shift. Opto-Electronic Engineering, 2007; 34(2): 100-104.

[16] Charilaos C, Joel A, Mathias L. Efficient methods for encoding regions of interest in the upcoming JPEG 2000 still image coding standard. IEEE Signal Processing Letters, 2000; 7(9): 247-249.

[17] Raphael G, Diego S C, Touradj E. New approach to JPEG 2000 compliant region of interest coding. SPIE, 2001; 4172: 267-275.

[18] Rema N R, Binu A O, Mythili P. Image compression using SPIHT with modified spatial orientation tress. Procedia Computer Science, 2015; 46: 1732-1738.

[19] Jcrome M S. Embedded image coding using zerotrees of wavelet coefficients. IEEE Tran on Signal Processing. 1993.

[20] Zhai L, Tang X M, Li L, Hong Z G. A new quality assessment index for compressed RS image. Geomatics and Information Science of Wuhan University, 2007; 32(10): 872-875. 